\title{
High flow nasal cannula oxygen therapy in adults with COVID-19 respiratory failure. A case report
}

\author{
Vasileios Karamouzos $^{1}$, Fotini Fligou1, Charalambos Gogos ${ }^{2}$, Dimitrios Velissaris ${ }^{2}$ \\ ${ }^{1}$ Intensive Care Unit, University Hospital of Patras, Rion; ${ }^{2}$ Internal Medicine Department, University Hospital of Patras, \\ Rion, Greece
}

\begin{abstract}
The novel corona virus (SARS-CoV-2) continuous to spread around the globe causing high mortality, tremendous stress on healthcare systems and an unprecedented disruption of everyday life with unpredictable socioeconomic ramifications. The disease is typically affecting the respiratory system and some patients will develop refractory hypoxemic respiratory insufficiency requiring mechanical ventilation. The role of non-invasive ventilation (NIV), high flow nasal cannula (HFNC) or continuous positive airway pressure devices (C-PAP) in the treatment of the 2019 corona virus disease (COVID-19) is not yet clear. We hereby report a case of a 44-year-old COVID-19 positive male patient
\end{abstract}

Correspondence: Vasileios Karamouzos, Intensive Care Unit, University Hospital of Patras, Rion, Greece.

Tel. +30.6942848933.

E-mail: vkaramouzos@hotmail.com

Key words: SARS-CoV-2; COVID-19; high flow nasal cannula.

Authors' contributions: All the authors made a substantive intellectual contribution. All the authors have read and approved the final version of the manuscript and agreed to be accountable for all aspects of the work.

Conflict of interest: The authors declared no potential conflicts of interest with respect to the research, authorship, and/or publication of this article.

Funding: The authors received no financial support for the research, authorship, and/or publication of this article.

Informed consent: Written informed consent was obtained from the patient and the relevant documents are available upon journals' request.

Received for publication: 17 April 2020.

Accepted for publication: 3 June 2020.

CCopyright: the Author(s), 2020

Licensee PAGEPress, Italy

Monaldi Archives for Chest Disease 2020; 90:1323

doi: 10.4081/monaldi.2020.1323

This article is distributed under the terms of the Creative Commons Attribution Noncommercial License (by-nc 4.0) which permits any noncommercial use, distribution, and reproduction in any medium, provided the original author(s) and source are credited. suffering from hypoxic respiratory failure that was successfully treated with high flow nasal cannula oxygen therapy in a negative pressure intensive care room. Although specific criteria for the use of high flow nasal canula devices in COVID-19 are not available at this time, clinicians could use this non-invasive modality as an alternative method of respiratory support in selected patients presenting with respiratory failure.

\section{Introduction}

Following the outbreak of the novel coronavirus (SARSCoV-2) last year in China, the virus has spread globally in more than 170 countries forcing the World Health Organization to declare a pandemic. More than two million confirmed cases were registered by April 16, 2020 raising the death toll from the 2019 novel coronavirus disease (COVID-19) over 130000. The clinical presentation of patients suffering from CoVid-19 can vary from asymptomatic to severe disease with multiorgan failure and commonly reported symptoms include fever, non-productive cough, shortness of breath, myalgia, and fatigue [1]. The main target of the virus is the respiratory system and some patients in the course of the disease will develop refractory hypoxemic respiratory insufficiency requiring mechanical ventilation. The role of noninvasive ventilation (NIV), high flow nasal cannula (HFNC) or continuous positive airway pressure devices (C-PAP) in the treatment of medium to severe Adult Respiratory Distress Syndrome (ARDS) from COVID-19 is not yet clear. Furthermore, health care safety issues are raised since virus dispersion may increase using high flow devices [2]. In this report we present a patient suffering from moderate ARDS due to COVID-19 that was successfully discharged from a negative pressure Intensive Care Unit (ICU) after treatment with HNFC.

\section{Case Report}

A 44-year-old male with a past medical history of hypertension controlled by angiotensin II receptor antagonist (valsartan $80 \mathrm{mg} \mathrm{qd}$ ) was admitted to the Emergency Department (ED) of a tertiary referral hospital in south-west Greece due to fever and mild respiratory distress. On admission the patient was alert, orientated and hemodynamically stable. He complained of a nonproductive cough accompanied by mild dyspnea on exertion and his axillary temperature was $38.3^{\circ} \mathrm{C}$. Arterial blood gas (ABG) analysis on room air confirmed hypoxemia $\left(\mathrm{PH}=7.42 ; \mathrm{PO}_{2}=63\right.$ $\mathrm{mmHg} ; \mathrm{PCO}_{2}=36 \mathrm{mmHg} ; \mathrm{HCO}_{3}=22.5 ; \mathrm{BE}=-1.5$ ) and chest aus- 
cultation revealed some crackles on the lower lobe of the right lung. No significant findings were noticed from the rest of the physical examination and his Chest X-ray on admission was unremarkable (Figure 1). Epidemiological anamnesis revealed that the patient had recently returned from a pilgrimage to the Middle East where COVID-19 cases were reported. A respiratory specimen was obtained and he was transferred to an isolated room in the Internal Medicine Department where he was treated with supplemental oxygen via nasal cannula (3 1/min), Ceftriaxone (2gr i.v. qd), Azithromycin (500 mg i.v. qd), and Oseltamivir (75 mg p.o. bid). The test for SARS-CoV-2 returned positive and a combination of lopinavir/ritonavir $(400 \mathrm{mg} / 100$ $\mathrm{mg}$ p.o. bid) and hydroxychloroquine (200 mg p.o. bid) were added to the treatment regimen.

On day 6 the patient's respiratory function suddenly deteriorated. He was treated with a Venturi face mask $\left(60 \% \mathrm{FiO}_{2}\right)$ and his $\mathrm{PaO}_{2} / \mathrm{FiO}_{2}$ ratio was 110 . Blood gas analysis revealed hypoxemia and respiratory alkalosis $\left(\mathrm{PH}=7.46 ; \mathrm{PO}_{2}=66 \mathrm{mmHg}\right.$; $\mathrm{PCO}_{2}=33 \mathrm{mmHg} ; \mathrm{HCO}_{3}=22.7 ; \mathrm{BE}=-0.8$ ) and a Chest CT scan showed extended scattered ground glass lesions in both lungs (Figures 2 and 3). Due to the significant deterioration of the patient's respiratory function he was transferred to a negative pressure isolation ICU room. The patient was alert, hemodynamically stable and a HFNC oxygen therapy trial was attempted. Initial $\mathrm{FiO}_{2}$ concentration and flow rate was $100 \%$ and $30 \mathrm{l} / \mathrm{min}$, respectively. The flow rate was gradually increased to $60 \mathrm{l} / \mathrm{min}$ and the $\mathrm{FiO}_{2}$ concentration was reduced to $60 \%$. The patient responded well to treatment, his $\mathrm{PaO}_{2} / \mathrm{FiO}_{2}$ ratio was 150 and it was decided to remain in HFNC therapy under close monitoring. The following days he remained stable and was instructed with the help of the physiotherapist to turn into the prone position three times a day leading to slow but progressive improvement of the oxygenation, and respiratory status. His $\mathrm{PaO}_{2} / \mathrm{FiO}_{2}$ ratio further increased above 250 and after 10 days he was successfully weaned from HFNC and was transferred to the internal medicine department.

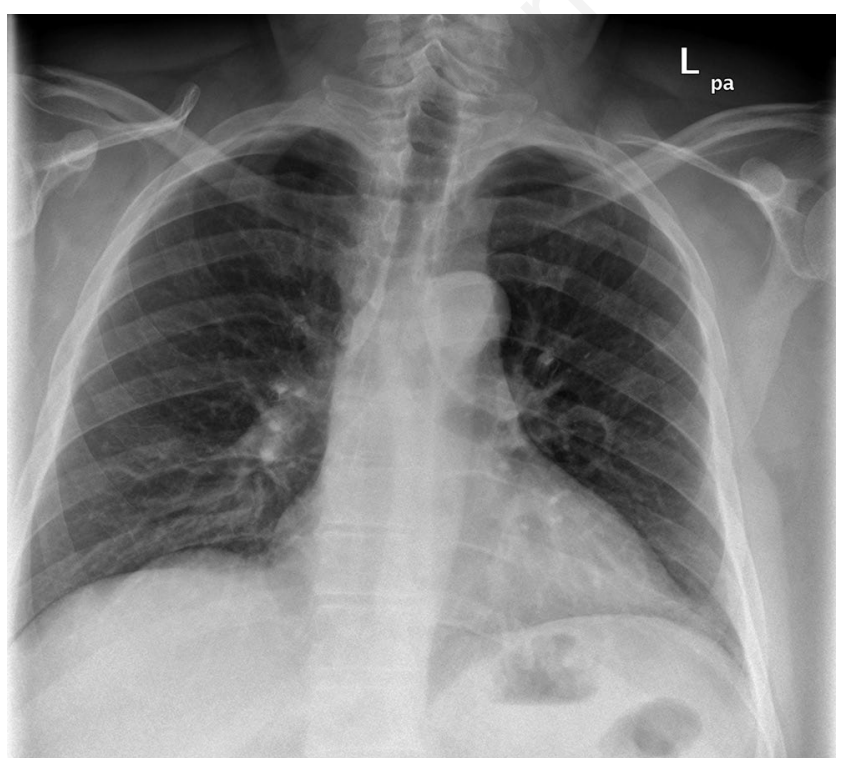

Figure 1. Chest X-ray on admission.

\section{Discussion}

SARS-CoV-2 is a positive-sense RNA virus, member of the coronavirus family, with a transmissibility similar to other respiratory viruses via large droplet transmission. Upon entering the host's organism, the virus binds to angiotensin-converting enzyme 2 (ACE 2) receptor on type II alveolar cells and intestinal epithelia, infiltrates the cell and starts replication. The main clinical findings include fever, upper and lower respiratory symptoms such as dyspnea, constitutional symptoms, and less commonly gastrointestinal symptoms (up to $10 \%$ ). Physical examination is generally non-specific. The typical disease course consists of an incubation period of 4 to 14 days; in severe cases, dyspnea is recognized around 6 days post exposure, admission to Hospital after 8 days and ICU admission after 10 days post exposure [1,3].

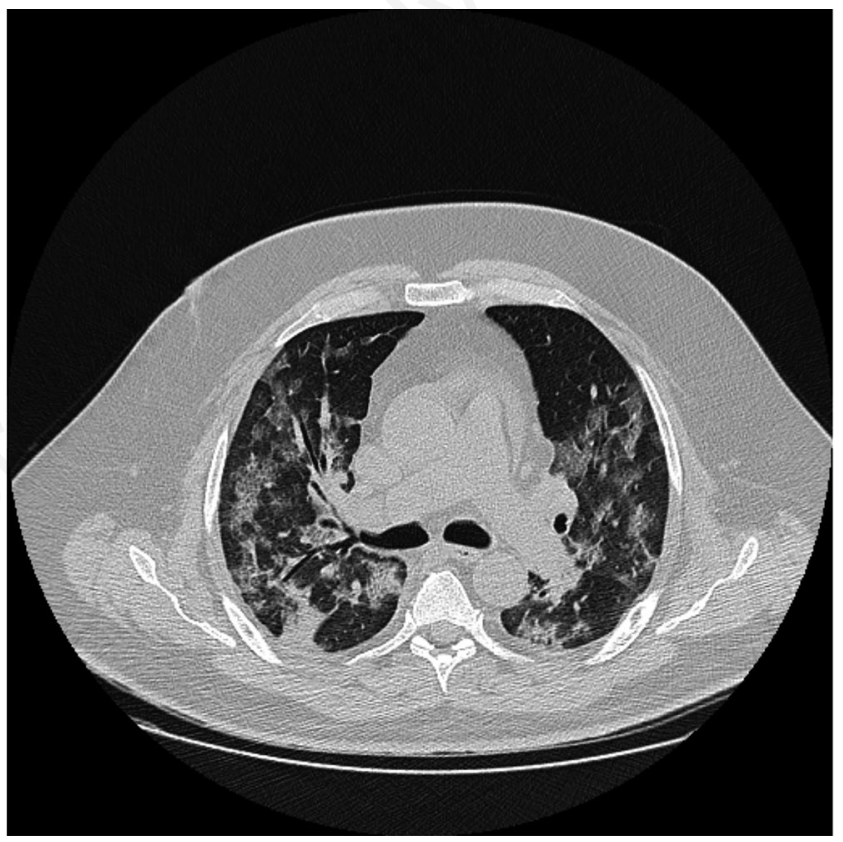

Figure 2. Chest computed tomography showing diffuse bilateral ground-glass opacities.

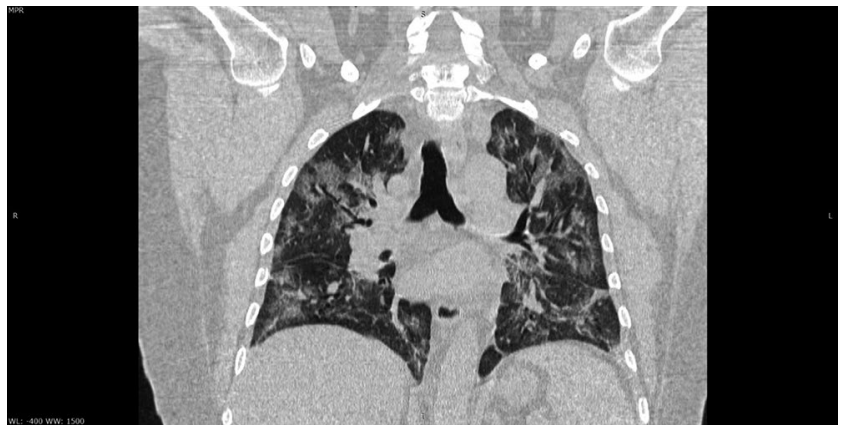

Figure 3. Coronal reconstruction in lung window showing diffuse bilateral infiltrations. 
Patients suffering from COVID-19 require oxygen therapy due to type 1 respiratory failure and as the disease progresses a difficult choice must be made between invasive and non-invasive ventilation. Nowadays a variety of different non-invasive systems with different patient interfaces offer us a multitude of therapeutic options. High-flow nasal cannula (HFNC) is a type of non-invasive oxygen therapy capable of delivering heated and humidified airoxygen mixtures at 0.21 to $1 \mathrm{FiO}_{2}$ and at flow rates up to $60 \mathrm{l} / \mathrm{min}$. HFNC is considered to have important physiological effects, such as reduction of patient's anatomical dead space, a PEEP effect and a constant fraction of inspired oxygen. Recently has gained a position in the field of respiratory support for critically ill adult patients as clinicians apply HFNC oxygen in a variety of situations, like hypoxemic respiratory failure, acute exacerbation of chronic obstructive pulmonary disease (COPD), post-extubation, pre-intubation, and to patients with end stage status. In regard to COVID19 , the true incidence of hypoxic respiratory failure is not clear, it seems, however, that about $14 \%$ of cases will develop severe disease requiring oxygen therapy, and $5 \%$ will require finally ICU admission and mechanical ventilation [4].

According to Surviving Sepsis Campaign (SSC) guidelines for the management of critically ill adults with COVID-19, in cases presenting with acute hypoxemic respiratory failure despite the conventional oxygen therapy, there is a weak recommendation for using HFNC over conventional oxygen therapy [5]. A systematic review and meta-analysis of 9 RCTs with 2,093 patients showed that the use of HFNC reduced the intubation rate compared to conventional oxygen therapy, but did not affect the risk of death or ICU length of stay [6-8]. In the COVID-19 pandemic, however, a possible reduction in intubation rate is crucial as medical resources worldwide are limited. Furthermore, the use of HFNC was better tolerated by the patients as they find it more comfortable in comparison to conventional oxygen therapy [9].

Comparing HNFC to NIV, SSC guidelines also recommend the use of HFNC over non-invasive positive pressure ventilation (NIPPV)(weak recommendation, LQE) [5]. A RCT comparing the two settings in patients with acute hypoxic respiratory failure, showed that HFNC resulted in reduced mortality at 90 days but did not significantly reduced the need for intubation, and a meta-analysis showed that HFNC decreased the need for intubation, yet without significantly reducing mortality or ICU length of stay $[6,9]$ Similar to conventional oxygen therapy, patients found HFNC more comfortable than NIPPV [9].

Most of the knowledge accumulated referring to the use of HFNC in respiratory tract viral infections is from patients with H1N1 influenza virus and SARS-CoV-1/MERS-CoV corona viruses. The experience of HFNC in patients with severe acute respiratory infection (SARI) has been described in a cohort of ICU patients admitted with SARI due to 2009 H1N1 Influenza A in a study by Rello et al. HFNC appeared to be an innovative and effective modality for the early treatment of adults with SARI [10]. Finally a recent small trial from China showed that from a total of 17 patients treated with HNFC, 7 (41\%) experienced treatment failure and among patients with $\mathrm{PO}_{2} / \mathrm{FiO}_{2}<200$ failure rates reached $63 \%$ [11].

Observational studies in patients with bacterial pneumonia, propose that HFNC does not seem to confer an increased risk of transmission of disease and the risk of bacterial environmental contamination is similar to that of conventional oxygen [12]. In patients with SARS treated with non-invasive methods the risk of transmission may be greater. Fowler at al. showed that nurses caring for patients with SARS receiving noninvasive positive-pressure ventilation may be at an increased risk (RR, 2.33; 95\% CI,
0.25 to $21.76 ; \mathrm{p}=0.5$ ) and the SSC guidelines suggests that all aerosol-generating procedures should be performed in a negative pressure room $[5,13]$. In any case patients receiving HFNC should be in a negative pressure room for maximum personnel safety and closely monitored in a setting where intubation can be facilitated immediately in the event of decompensation.

In our case, we decided to try a non-invasive modality due to the patient's single system involvement. Decisive factors in choosing HNFC over NIV/CPAP therapy were the fact that our patient was suffering from respiratory failure type I and the tolerability profile of HFNC. As previously reported, awake prone positioning can significantly improve oxygenation and pulmonary heterogeneity [14]. HNFC allowed our patient to turn into the prone position 3 times a day, a maneuver which also helped in improving oxygenation.

\section{Conclusions}

HFNC oxygen delivery could be an additional option and should be available in our armamentarium as an alternative method of respiratory support in patients suffering from COVID-19. Although specific criteria for commencing or stopping HFNC therapy are not available at this time, clinicians could use this noninvasive modality in selected COVID-19 patients suffering from respiratory failure.

\section{References}

1. Guan W-J, Ni Z-Y, Hu Y, et al. Clinical Characteristics of coronavirus disease 2019 in China. N Engl J Med 2020;382:1708 20. doi: 10.1056/NEJMoa2002032.

2. Guan L, Zhou L, Zhang J, et al. More awareness is needed for severe acute respiratory syndrome coronavirus 2019 transmission through exhaled air during non-invasive respiratory support: experience from China. Eur Respir J 2020;55:2000352. doi: 10.1183/13993003.00352-2020.

3. Huang C, Wang Y, Li X, et al. Clinical features of patients infected with 2019 novel coronavirus in Wuhan, China. Lancet 2020;395:497-506.

4. Wu Z, McGoogan JM. Characteristics of and important lessons from the coronavirus disease 2019 (COVID-19) outbreak in China: Summary of a report of 72314 cases from the Chinese Center for Disease Control and Prevention. JAMA 2020. doi: 10.1001/jama.2020.2648.

5. Oczkowski S, Levy MM, Derde L, et al. Surviving Sepsis Campaign: Guidelines on the management of critically ill adults with coronavirus disease 2019 (COVID-19). Intensive Care Med 2020;46:854-87. doi: 10.1007/s00134-020-06022-5.

6. Ni Y-N, Luo J, Yu H, et al. The effect of high-flow nasal cannula in reducing the mortality and the rate of endotracheal intubation when used before mechanical ventilation compared with conventional oxygen therapy and noninvasive positive pressure ventilation. A systematic review and meta-analysis. Am J Emerg Med 2018;36:226-33.

7. Ou X, Hua Y, Liu J, et al. Effect of high-flow nasal cannula oxygen therapy in adults with acute hypoxemic respiratory failure: a meta-analysis of randomized controlled trials. CMAJ 2017;189:E260-7.

8. Rochwerg B, Granton D, Wang DX, et al. High flow nasal cannula compared with conventional oxygen therapy for acute 
hypoxemic respiratory failure: a systematic review and metaanalysis. Intensive Care Med 2019;45:563-72.

9. Frat JP, Thille AW, Mercat A, et al. High-flow oxygen through nasal cannula in acute hypoxemic respiratory failure. N Engl J Med 2015;372:2185-96.

10. Rello J, Pérez M, Roca O, et al. High-flow nasal therapy in adults with severe acute respiratory infection: a cohort study in patients with 2009 influenza A/H1N1v. J Crit Care 2012;27: 434-9.

11. Wang K, Zhao W, Li J, et al. The experience of high-flow nasal cannula in hospitalized patients with 2019 novel coronavirusinfected pneumonia in two hospitals of Chongqing, China. Ann Intensive Care 2020;10:37. doi: 10.1186/s13613-020-00653-z.
12. Leung CCH, Joynt GM, Gomersall CD, et al. Comparison of high-flow nasal cannula versus oxygen face mask for environmental bacterial contamination in critically ill pneumonia patients: a randomized controlled crossover trial. J Hosp Infect 2019;101:84-7.

13. Fowler RA, Guest CB, Lapinsky SE, et al. Transmission of severe acute respiratory syndrome during intubation and mechanical ventilation. Am J Respir Crit Care Med 2004;169: 1198-202.

14. Sun Q, Qiu H, Huang M, Yang Y. Lower mortality of COVID19 by early recognition and intervention: experience from Jiangsu Province. Ann Intensive Care 2020;10:33. doi: 10.1186/s13613-020-00650-2. 